\title{
ANÁLISIS DE LA PERSPECTIVA DE GÉNERO EN LOS PLANES DIRECTORES DEL DEPARTAMENTO DE SALUD DE LA GENERALITAT DE CATALUNYA
}

\author{
$M^{a}$ LUISA PANISELlO CHAVARRIA, \\ INMA PASTOR GOSALBEZ Y M ${ }^{\mathrm{a}}$ LUISA MATEU GIL \\ Universidad Rovira i Virgili \\ AnNa PANisello TAFalla \\ Especialista en Medicina Familiar y Comunitaria. \\ Hospital Verge de la Cinta de Tortosa (Tarragona)
}

Recibido/15/07/2011

Aceptado/25/09/2011

\section{Resumen}

La perspectiva de género entra a formar parte de las políticas públicas para paliar las desigualdades entre hombres y mujeres. Los elementos que consideramos que tendrían que estar presentes en las políticas públicas con perspectiva de género son: la visibilidad de las mujeres, la participación paritaria en los organismos determinados, el lenguaje que no sea androcéntrico ni sexista, la transversalidad y los determinantes de género como construcción social. El objetivo de nuestro trabajo es analizar si la perspectiva de género está presente en los Planes Directores del Departamento de Salud de la Generalitat de Catalunya.

Palabras clave: Perspectiva de género, Planes Directores, Sistema Sanitario Catalán.

\begin{abstract}
Gender perspective becomes part of public policies to avoid inequalities between men and women. The elements that we believe would have to be present in public policies with gender perspective are: the visibility of women, equal participation, not andocentric or sexist language, transverse and gender determinants as social construction.

The aim of our study is to analyze whether the gender perspective is present in the Master Plan of the Department of Health of the Generalitat de Catalunya.
\end{abstract}

Keywords: Gender perspective, sanitary system, Master plans.

Feminismo/s 18, diciembre 2011, pp. 135-154 


\section{Introducción}

El análisis de género ha permitido poner en evidencia el androcentrismo de la ciencia, la lengua, la historia,... y poner de manifiesto la construcción social del género. Es a partir de este análisis y cuando las mujeres acceden a la formación, entrando a formar parte de los poderes públicos, no de forma anecdótica sino como miembros de una sociedad formada por hombres y mujeres, cuando se cuestiona la sociedad androcéntrica y los poderes públicos empiezan a formular políticas públicas introduciendo la perspectiva de género.

Las políticas públicas con perspectiva de género, como procesos por los que son elaborados e implementados programas de acción pública, es decir dispositivos políticos-administrativos coordinados en torno a objetivos explícitos, según Guzmán Virginia, ${ }^{1}$ buscan corregir la desigualdad que se genera en el sistema de género favoreciendo la equidad entre los hombres y las mujeres. »La institucionalización de las políticas públicas de género es el proceso mediante el cual una nueva práctica se incorpora a las instituciones del Estado, se hace estable, se reitera en el tiempo y por lo tanto permite, seguimiento y evaluación». ${ }^{2}$

Cada actuación del Estado puede tener un impacto diferente en la situación de los hombres y las mujeres y producir aunque sea de manera indirecta situaciones que perjudiquen la equidad de género de ahí la necesidad de introducir la dimensión de género en todas las actuaciones de las instituciones del Estado.

En cuanto a la interrelación de género y política hay que señalar que:

- La política está determinada por el sistema de género, actividad mayoritariamente masculina

- El sistema de género se mantiene y se reproduce por la intervención política.

1. GuZMAn,Virginia.La institucionalidad de género en el estado: nuevas perspectivas de análisis. Chile. CEPAL, Série Mujer y Desarrollo, núm 3, 2009.

2.AstelarRA, Judith, Veinte años de politicas de igualdad. Madrid. Ediciones Cátedra. 2005.

Feminismo/s 18, diciembre 2011, pp. 135-154 
- Cada actuación del estado puede tener un impacto diferente en la situación de los hombres y las mujeres y producir aunque sea de manera indirecta situaciones que perjudiquen la equidad de género. Hay que introducir la dimensión de género en todas las actuaciones de las instituciones del Estado. ${ }^{3}$

\section{Perspectiva de género en el sistema sanitario}

La Ley General de Sanidad de España (Ley 14/1986 del 25 de abril) tiene por objeto la regulación general de todas las acciones que permitan hacer efectivo el derecho a la protección de la salud, establece las bases de un modelo de ordenación sanitaria que se construye mediante la creación del Sistema Nacional de Salud, configurado por el conjunto de los Servicios de Salud de las Comunidades Autónomas, por lo tanto tiene un carácter descentralizador.

Así lo recoge la Ley 15/1990 de 9 de julio de Ordenación Sanitaria de Cataluña, siendo una de las finalidades de esta ley: «La atención integral de la salud que comprende: la promoción de la salud, la prevención de la enfermedad así como las acciones curativas y rehabilitadoras necesarias, colaborando en la reinserción social y garantizar la salud como un derecho inalienable».

En Cataluña se crea el Servei Català de la Salut como ente para llevar a cabo la organización y ordenación del Sistema Sanitario Catalán. En el artículo 62, nos habla del Plan de Salud de Cataluña y dice:

Las líneas directivas y de desarrollo de las actividades, los programas y los recursos del Servicio catalán de la salud para alcanzar sus fines constituyen el Plan de Salud de Cataluña. El Plan de Salud es el instrumento indicativo y el marco de referencia para todas las actuaciones públicas en la materia, en el ámbito de la Generalidad de Cataluña». (Art.62, apar.1)

Los planes de salud articulan las políticas de salud y se incluyen los principios, valores, objetivos y acciones a desarrollar durante el periodo de vigencia, definiendo lo que se considera problema de salud y la priorización de los mismos. En Cataluña se inician los planes de salud con el Documento Marco en 1991, a partir de esta fecha tenemos los siguientes planes (93-95, 96-98,992001,2002-2005 y Plan de Salud en el horizonte 2010). ${ }^{4}$

Los principales instrumentos de planificación estratégica del Departamento de Salud de la Generalitat de Cataluña son: El Plan de Salud, los Planes Directores y el Mapa Sanitario, Sociosanitario y de Salud Pública. El Plan de

3. Ibid. p. 31.

4. http://www.gencat.cat/salut/depsalut/html/ca/plasalut/index.html, última consulta $1-3-2011$. 
Salud determina las prioridades de salud que se estructuran como punto de referencia para la visión estratégica del Mapa. Los Planes Directores operativizan las prioridades del plan de salud, en base al artículo 64 de la Ley 15/1990 de 9 de julio, de ordenación sanitaria de Cataluña que establece que corresponde al Departamento de Salud formular los criterios generales de la planificación sanitaria y también fijar los objetivos, los índices y los niveles básicos a alcanzar en las materias que se incluyen en el Plan de Salud de Cataluña. El primer Mapa Sanitario de Cataluña se presentó en 1980 y el último publicado se aprobó por acuerdo de Gobierno GOV/2008 de 20 de mayo.

El Departamento de Salud priorizó seis materias, en base al análisis de la salud, los servicios sanitarios y del contexto socio económico del país, como planes directores para el periodo 2005-2007, que fueron el de la inmigración, el sociosanitario, el de salud mental y adicciones, la oncología, las enfermedades del aparato circulatorio, y la investigación en ciencias de la salud, aprobados por Decreto de 28 de febrero de 2006 (DOGC núm. 4584 de 2-3-2006). Por acuerdo de Gobierno el 18 de julio de 2006, se aprueban los Planes Directores 2005-07 con una vigencia de 3 años (DOGC núm. 4689-02/08/2006). ${ }^{5}$ En estos Decretos, el artículo 2 nos define los objetivos de cada Plan y en el apartado 2.2 señala:

En el desarrollo de estas funciones se hará el seguimiento y la validación del uso de la variante de género, de forma que se tengan en cuenta las especificidades de salud de las mujeres con el objeto de dar respuesta y ofrecer una atención integral ante sus necesidades.

En el artículo 7 nos habla del Consejo Asesor y en el apartado 7.2 señala:

En la composición del Consejo Asesor se procurará alcanzar la presencia de un número de mujeres que represente un mínimo del 50\% del total de personas miembros de este órgano colegiado.

La IV Conferencia Mundial de las Naciones Unidas sobre las Mujeres que tuvo lugar en Beijing (Septiembre, 1995) marca la línea que debe orientar toda acción de gobierno analizando los efectos desde el punto de vista del género de todas las políticas y programas.

La Organización Mundial de la Salud (OMS) elaboró en 2002, el documento que insta a los países miembros a elaborar estrategias para promover la integración del género en los sistemas de salud y nos relata la política de la OMS en materia de género: Integración de las perspectivas de género en la labor de la OMS. En la declaración introductoria Gro Harlem Brundtland, Directora

5. http://www.gencat.cat/salut/depsalut/html/ca/dir2154/index.html. Consultado 25-9-2010.

Feminismo/s 18, diciembre 2011, pp. 135-154 
General, señala que la integración de las perspectivas de género en la acción sanitaria obedece a un criterio justo de salud pública.

El documento refiere que la política de la Organización será velar para que todas las investigaciones, las políticas, los proyectos, los programas y las iniciativas en las que participe la OMS, incorpore las cuestiones de género.

Entre las metas y objetivos destacamos:

- Promover la equidad y la igualdad entre hombres y mujeres a lo largo de toda la vida

- Proporcionar información cualitativa y cuantitativa sobre la influencia del género en la salud y la atención de salud

- Promover la integración de los asuntos de género en los sistemas de salud.

En la Declaración del Milenio de Naciones Unidas y en otros acuerdos internacionales se reconoce la importancia de la igualdad de derechos entre hombres y mujeres y el derecho de todos a vivir sin sufrir discriminaciones en ninguna esfera de la vida, incluyendo el acceso a la atención de salud.

Recogiendo el sentir de estas Instituciones, en el estado español, la Ley Orgánica 3 / 2007, de 22 de marzo, para la Igualdad Efectiva de Mujeres y Hombres. (BOE núm. 71 Viernes 23 marzo 2007) en el Artículo 27, trata de la integración del principio de igualdad en la política de salud, y el Sistema Sanitario debería orientar sus acciones integrando la perspectiva de género para alcanzar la equidad y la igualdad entre hombres y mujeres.

Para alcanzar este fin, a nivel internacional, estatal y autonómico se han desarrollado guías en relación a la incorporación de la perspectiva de género tanto en las políticas públicas como en el ámbito de la salud y la investigación. ${ }^{6}{ }^{7}$

\subsection{Objetivo del estudio}

El objetivo de nuestro trabajo es analizar si la perspectiva de género está presente y de que manera se incorpora, en los Planes Directores del Departamento

6. Garcia Calvente Ma del Mar, Jiménez Rodrigo MáLuisa, Martínez Morante Emilia. Informe de revisión de guias sobre la incorporación de la perspectiva de género a las plíticas de investigación en salud. Observatorio de Salud de la Mujer (OSM). Dirección general de la agencia de Calidad del Sistema nacional de Salud. Secretaria General de Sanidad. Ministerio de Sanidad y Consumo. Escuela Andaluza de Salud Pública. 2005.

7. VELASCO ARIAS Sara. Recomendaciones para la práctica del enfoque de género en programas de salud. Observatorio de Salud de la Mujer (OSM). Dirección general de la agencia de Calidad del Sistema nacional de Salud. Secretaria General de Sanidad. Ministerio de Sanidad y Consumo Madrid 2008. 
de Salud de la Generalitat de Cataluña, siendo nuestra hipótesis de partida que la voluntad política de integrar la perspectiva de género en el Sistema Sanitario esta presente, pero esta no queda reflejada en la redacción incorporándose parcialmente en el diseño

\subsection{Metodología}

Se han analizado los Planes Directores para el período 2005-2007, que en la actualidad siguen estando en vigor. Estos son el de la inmigración, el sociosanitario, el de salud mental y adicciones, la oncología y las enfermedades del aparato circulatorio. Profundizaremos en este último por ser la primera causa de muerte en las mujeres. En el artículo. 2.2 de todos los Planes, señala:

En el desarrollo de las funciones se hará seguimiento y validación del uso de la variante de género, de forma que se tengan en cuenta las especificidades de salud de las mujeres con el objetivo de dar respuesta y ofrecer una atención integral ante sus necesidades.

Para realizar este análisis nos hemos basado en la «Guía para facilitar la incorporación de la perspectiva de género en los Planes Integrales de salud» de la Consejería de Salud (Junta de Andalucía, 2008), adaptándola a los objetivos de nuestro estudio. ${ }^{8}$

Los elementos que consideramos que deberían estar presentes en el diseño de una política pública con perspectiva de género son:

- Visibilidad: Los datos deben estar disgregados por sexos para posteriormente realizar el análisis de las diferencias encontradas con perspectiva de género.

- Participación: En las comisiones del Plan se procurará la paridad (Artículo 7 de los respectivos Planes). Revisaremos si los nombramientos de los equipos, planes directores, colaboradores / as del departamento, consejos asesores, comisión permanente, grupos de trabajo, órganos de participación tienen en cuenta paridad.

- Lenguaje y imágenes: Su uso no debe ser androcéntrico ni sexista

- Determinantes de género: Verificar si los elementos propios de género están presentes en la redacción de los diferentes Planes.

- Transversalidad: Si los Planes tienen en cuenta actuaciones transversales y con otras instituciones en relación al género

8. Amo Alonso, Mercedes. Guía para facilitar la incorporación de la perspectiva de género en los Planes Integrales de salud. Sevilla. Consejeria de Salud. 2008. 


\section{Analisis de la perspectiva de género de los planes directores}

\subsection{Análisis de la perspectiva de género: plan director de inmigración en el} ámbito de la salud

El Plan Director de Inmigración en el ámbito de la salud y su Consejo Asesor se crea por decreto 40/2006 de 14 de marzo y se publica en octubre de $2006 .{ }^{9}$ La misión del Plan Director de Inmigración (PDI) es la de mejorar la salud de la población inmigrada mediante la definición de un modelo de atención y organización de los servicios que permita al sistema de salud catalán hacer frente a la nueva situación planteada por el fenómeno de la inmigración.

En relación a la visibilidad de las mujeres en el redactado del Plan, en la descripción de la situación aparecen algunos datos desglosados por sexo. De las diferencias encontradas no se realiza el posterior análisis con perspectiva de género.

Los objetivos son genéricos y las mujeres sólo quedan reflejadas en los que hacen referencia a la salud sexual y reproductiva.

Al referirse a los contenidos de la formación la única referencia que se hace a la mujer inmigrante es la salud reproductiva.

En cuanto a la participación en el equipo del Plan Director hay una mayoría de mujeres ( $71,4 \%$ frente $28,5 \%$ de hombres), la relación se invierte con los colaboradores del Departamento de Salud (38, 1\% de mujeres frente 61,8\% de hombres). En cuanto a la composición de los grupos de trabajo según el sexo de los participantes hay que señalar que en los de salud internacional y sistemas de información la totalidad de los participantes son hombres (100\%) mientras que en las de mediación $(68,4 \%$ son mujeres) y acogida $(70,3 \%$ son mujeres).

En relación al lenguaje, en general sí que se tiene en cuenta el no invisibilizar a las mujeres, aunque hay veces que utiliza el masculino para referirse a los dos géneros (niños, hijos, mediadores,....). La imagen que utiliza en la portada y en el folleto es el de una doctora auscultando a una niña.

En relación a la perspectiva de género, no referencia bibliografía relacionada con el género pero sí material editado por el Instituto Catalán de las Mujeres (ICD). No se tiene en cuenta que los determinantes de salud de los hombres y las mujeres son diferentes y por tanto también las necesidades de salud. Se limita a la salud sexual y reproductiva en relación a las mujeres, formulando sólo objetivos en relación a la salud materno

9. Pla Director d'Immigració en l'Àmbit de la Salut. Direcció General de Planificació i Avaluació 2006. Barcelona. 
infantil, enfermedades de transmisión sexual y cáncer de cuello de útero. Se formulan objetivos específicos que impulsan la búsqueda de indicadores sensibles al género en las interrupciones voluntarias del embarazo, embarazos en adolescentes, mutilación genital femenina, salud sexual y reproductiva.

Las propuestas de actuaciones para alcanzar los objetivos explícitamente no tienen en cuenta la perspectiva de género ni disminuir los sesgos de género en el conocimiento existente.

El Plan promueve acciones para lograr la formación con perspectiva de género en todos los perfiles profesionales.

Cabe destacar que en lo referente a las mujeres se limita a la salud sexual y reproductiva y las mutilaciones genitales femeninas.

Se han revisado otros documentos en relación a las mujeres emigradas ya que es el sector de la población donde la invisibilidad por razón de género es más evidente. En relación a la transversalidad este Plan no contempla la integración de la perspectiva de género de forma explícita como uno de los ejes en todos los apartados del Plan Director, pero comparando con los otros Planes podemos decir que es el Plan que más trabaja de forma transversal con otros Departamentos y sobre todo con el ICD. En concreto el Plan Integral de Acogida (PIA) que forma parte del Plan Director, no hace referencia al género en ningún objetivo. Pero nos habla de la elaboración de material de apoyo e información de acogida con el $\mathrm{ICD}^{10}$. Esto constituye un ejemplo de trabajo transversal en materia de género. Dentro de la colección «Cuadernos del Instituto» del ICD el $\mathrm{n}^{\circ} 5$ está dedicado a las mujeres emigradas. Este cuaderno nos señala la situación de las mujeres emigradas que son mujeres pobres, de otro país, otra etnia y trabajadoras, estos elementos influyen en la feminización de la pobreza, hay un techo de cristal para estas mujeres. ${ }^{11}$

También refiere que cuando se habla de inmigración se olvida del porcentaje de mujeres emigradas y que una vez más estamos ante una nueva invisibilización de las mujeres. En cuanto a la salud destaca la necesidad de la incorporación de la temática del proceso migratorio, las enfermedades específicas (depresiones,...) y la importancia que tendría tratar el tema desde una perspectiva de género. Siguiendo las iniciativas del ICD, cabe destacar que en el «Plan de acción de gobierno en políticas de mujeres (2008-2011)» se contempla las acciones en relación a la inmigración que tiene que llevar a cabo el Departamento de Salud.

10. http://www20.gencat.cat/portal/site/icdones. Consultado el 9-10-2010.

11. SIPI, Remei. ARAYA, Mariel. Les dones migrades. Generalitat de Catalunya. Institut Català de les Dones. Barcelona. Quaderns de l'Institut 5. (2007). 
Otro ejemplo de esta transversalidad lo constituye las actuaciones formativas del ICD, en el catálogo de talleres, uno hace referencia a «Mujeres y salud: espacios de participación» A si mismo en el tríptico de bienvenida a Cataluña donde explica los derechos de las mujeres en referencia a la salud. «Derecho a la salud y a decidir sobre el propio cuerpo», nos habla del derecho a la salud, de la planificación, los embarazos y el parto. En cuanto a Salud e inmigración el Departamento de Salud tiene el listado de protocolo y guías.

Mención especial es todo el tema de la mutilación genital femenina, que para el gobierno de la Generalitat del momento fue objeto de especial atención. Como ejemplo hay que citar una publicación del Departamento de Salud sobre el tema, la prevención y atención. Como política transversal, se ha revisado el Pacto Nacional por la Inmigración (PNI). «Un pacto para vivir juntos y juntas». Firmado por los Sindicatos, representantes de la Mesa de ciudadanía e inmigración y por todos los grupos parlamentarios a excepción del Partido Popular, en Barcelona en diciembre de 2008.

Entre los principios del PNI figura:

- Incorporar la perspectiva de género durante todo el proceso y como herramienta de análisis y de planificación.

El PNI apuesta por mantener la coordinación entre los entes locales y los servicios de salud y la voluntad comunitaria y participativa de trabajo en el territorio para llevar a cabo los proyectos elaborados desde el Plan Director de Inmigración en el ámbito de la salud.

\subsection{Análisis de la perspectiva de género: plan director sociosanitario (PDSS)}

El Plan Director Sociosanitario y su Consejo Asesor se crea por Decreto 39/2006 de 14 de marzo. Diario oficial de la Generalidat Núm 4594. El Plan Director considera la atención sociosanitaria como aquella que se dirige a los colectivos referidos en el Plan: personas mayores, personas con enfermedad de Alzheimer u otras demencias, pacientes con otras enfermedades neurológicas que pueden cursar con discapacidad y personas en situación de final de la vida, haciendo especial mención a la atención prestada a las personas en situación de mayor riesgo y / o vulnerabilidad en procesos, crónicos o agudos, con diferentes grados de discapacidad y cualquier etapa del ciclo vital. ${ }^{12}$

En relación a la visibilidad de las mujeres dentro del Plan, en la descripción de la situación hay datos disgregados por sexo que nos muestran las diferencias que existen respecto a la morbimortalidad, estilos de vida, dependencias

12. Pla Director Socioanitario. Direcció General de Planificació i Avaluació 2006. Barcelona. 
en relación al sexo. No hay un análisis posterior de las diferencias de estos datos, sólo se limita a describirlas. En cuanto a la participación en el equipo del Plan Director hay una mayoría de mujeres (71,4\% frente 28,5\% de hombres), la directora del PDSS es una mujer. La proporción de los colaboradores del Departamento de Salud es de 44\% de mujeres frente 56\% de hombres. En cuanto a la composición de los grupos de trabajo en relación al sexo de los participantes, en el caso del grupo de Demencia y Geriatría el 53,8\% son mujeres y en el grupo de Cuidados Paliativos son mujeres el 56,2\%.

En relación al lenguaje, en general este Plan utiliza el masculino para referirse a los dos géneros (cuidador, enfermo, médicos, enfermeros, trabajadores sociales,...) La imagen que utiliza en el folleto es de un señor mayor con una niña (se supone el abuelo con la nieta).

En relación a la transversalidad, no contempla la integración de la perspectiva de género, de forma explícita como uno de los ejes en todos los apartados del Plan director. En relación a la perspectiva de género no cita bibliografía al respecto. La descripción de la situación no se traduce en ninguna propuesta ni objetivo para disminuir el sesgo relacionado con el género. La única propuesta donde aparecen las mujeres es en el hecho de ser cuidadoras (situación relacionada con el rol tradicional). No hay ninguna propuesta de investigación en relación al género y el problema de salud determinado por el Plan Director.

Tampoco queda constancia de la promoción, de forma explícita, de acciones para lograr la formación con perspectiva de género en relación al PDSS, para ningún perfil profesional.

\subsection{Análisis de la perspectiva de género: plan de salud mental y adicciones} (PDSMA)

El Plan Director de Salud Mental y Adicciones y su Consejo Asesor, se crea por el Decreto 30/2006 de 28 de febrero. (DOGC núm. 4584).

La salud mental ha sido declarada recientemente una prioridad en el seno de la Unión Europea (Declaración de Helsinki, enero de 2005). Esta declaración señala que hay que enfocar los graves problemas de salud mental desde las políticas públicas con una perspectiva integradora. El riesgo de mala salud mental es más elevado en las mujeres en todos los grupos de edad y va aumentando a medida que bajamos en la clase social. La OMS define la salud mental (SM) como un estado de bienestar en el que el individuo es consciente de sus capacidades, puede enfrentarse a las exigencias normales de la vida y trabajar de forma productiva y fructífera, y es capaz de contribuir a su comunidad. La misión del Plan Director de salud mental y adicciones es decidir, planificar, 
ejecutar y evaluar las acciones más efectivas y los recursos más apropiados para la mejora de la salud mental de la población. Los proyectos e intervenciones definidos en este Plan apuntan a los siguientes objetivos del Plan de Salud de Cataluña en el horizonte 2010:

A. Problemas de salud mental

B. Consumo excesivo de alcohol

C. Consumo de drogas no institucionalizadas ${ }^{13}$

En relación a la visibilidad, como primera dimensión de la perspectiva de género, en el análisis de la situación, el Plan disgrega los datos de morbilidad y mortalidad por sexo, esta disgregación refleja que los trastornos mentales son un problema de salud donde las diferencias en las prevalencias de los problemas son visibles en relación al sexo, pero esta evidencia no va seguida de un análisis de las posibles causas de esta diferencia.

Las propuestas de actuación y los objetivos del plan director no tienen en cuenta los resultados del análisis de los datos disgregados ni la perspectiva de género. Dentro del objetivo: «Promoción de la salud mental y prevención de los trastornos mentales y las adicciones» nos señala que hay que incorporar la perspectiva de edad y género, pero después no se formula ningún objetivo específico que impulse la búsqueda de indicadores sensibles al género. En el objetivo operativo 3.3 en relación a la salud mental de los adultos contempla la elaboración de un proyecto de prevención y detección precoz de mujeres maltratadas en el marco interdepartamental coordinado por el ICD, esto sería otro ejemplo de transversalidad.

Sólo en el objetivo operativo de «Promocionar la investigación clínica» entre los proyectos de investigación figura el «Proyecto de investigación sobre los maltratadores de género, abuso infantil».

En los proyectos a priorizar, en uno hace mención a incluir programas de prevención y detección precoz en las unidades integrales de violencia de género y en otro de incorporar los aspectos de atención psiquiátrica y psicológica a las mujeres maltratadas.

En relación a la participación, en el registro de actores (así lo denomina el Plan) del Plan, la presencia de mujeres es importante (equipo del Plan 60\% de mujeres). Debemos señalar que en la composición de los grupos de trabajo que están formados en relación a las etapas del ciclo vital (infantil, adolescencia, adultos y ancianos), las mujeres forman parte de forma mayoritaria en el

13. Pla Director de Salut Mental i addiccions de Catalunya. Direcció General de Planificació i Avaluació 2006. Barcelona. 
grupo de trabajo de infantil $(73,6 \%)$, el grupo de adolescentes $(64,8 \%)$ y en el de las personas mayores $(52,6 \%)$ pero en el grupo de adultos la presencia de mujeres es menor $(38,4 \%)$.

En relación al lenguaje utilizado no visibiliza igual a hombres y mujeres. La imagen visual de la portada y del folleto refleja la presencia de mujeres y hombres en diferentes etapas del ciclo vital.

No hay referencias explícitas en la bibliografía utilizada que haga mención a la relación del género con la salud mental y sus problemas así como con las adicciones

\subsection{Análisis de la perspectiva de género: plan director de oncología}

Los Objetivos generales que se marca el Plan director de oncología son:

1. Reducir la incidencia y la mortalidad en cáncer en Cataluña.

2. Mejorar la calidad de vida en todo el proceso.

3. Mejorar la accesibilidad, garantizando el diagnóstico precoz y un tratamiento apropiado y de calidad.

4. Mejorar la información y comunicación entre ciudadanos, pacientes, familiares, profesionales, gestores y políticos. ${ }^{14}$

En relación a la visibilidad, los datos referentes a la morbi-mortalidad están disgregados por sexo pero no así las referentes a los factores de riesgo ni los ingresos hospitalarios. No se realiza ningún análisis de los datos para buscar relaciones con el género.

En relación a la participación, las personas que participan en la elaboración del Plan (Actores del Plan), el Equipo del Plan (42,8\% de mujeres) y los colaboradores del departamento ( $41,3 \%$ de mujeres) se puede hablar de paridad en la composición, no así en el Consejo Asesor (17,6\% de mujeres frente a $82,3 \%$ de hombres) ni en la comisión permanente (25\% de mujeres frente a $75 \%$ de hombres).

Observamos gran variación en cuanto a la composición por sexo en los grupos de trabajo, el de psico-oncología está formado por un 83,3\% de mujeres y en el del cuello uterino 76,1 de mujeres (forman parte de este grupo una representante del ICD, una de «La Xarxa de Dones per la Salut» y una de la coordinadora de Usuarios de la Salud), frente al de radioterapia que son un $11 \%$ las mujeres y el de anatomía patológica que el 100\% son hombres.

14. Pla Director d'Oncologia. Direcció General de Planificació i Avaluació 2006. Barcelona.

Feminismo/s 18, diciembre 2011, pp. 135-154 
El lenguaje empleado en la redacción del plan utiliza generalmente el masculino para referirse a los dos sexos.

En cuanto a las imágenes se constata que en la portada del Plan y del tríptico aparece una mujer de mediana edad.

En la bibliografía referenciada no aparece ninguna cita de ninguna publicación en referencia al cáncer y el sexo / género.

En relación a la transversalidad, no se contempla la integración de la perspectiva de género, de forma explícita como uno de los ejes, en todos los apartados, del plan director. Pero hay una representante del ICD, una de red de Mujeres por la Salud y una de la coordinadora de Usuarios de la Salud en un grupo de trabajo. En la formulación de objetivos no se tiene en cuenta que los determinantes de salud y los factores de riesgo son diferentes para los hombres y las mujeres. En los objetivos del Plan no se tiene en cuenta la relación del género con el cáncer de mama, que es el más frecuente en las mujeres y que causa más mortalidad.

Tampoco se promueven acciones para lograr la formación con perspectiva de género en todos los perfiles profesionales.

\subsection{Análisis de la perspectiva de género: plan director de las enfermedades del aparato circulatorio (PDMAC)}

En la presentación de éste se indica que el Plan de las enfermedades del aparato circulatorio (PDMAC) es el instrumento de información, estudio y propuesta mediante el cual el Departamento de Salud determina las líneas directrices para impulsar, planificar, coordinar y evaluar las actuaciones a desarrollar en el ámbito de la promoción de la salud de las enfermedades del aparato circulatorio (enfermedades cardiovasculares y vasculares cerebrales). La prevención, el tratamiento y la rehabilitación, tienen el objetivo de mejorar la calidad de vida de los afectados. ${ }^{15}$ El PDMAC reparte sus objetivos, estrategias y prioritarios en dos Planes: Plan Director de enfermedad cerebro-vascular y el Plan Director de enfermedad cardiovasculares Como ya hemos se ha indicado, el estudio que da lugar a este artículo ha profundizado en el Plan Director de las Enfermedades cardiovasculares.

En el momento de redactar el Plan Director numerosas publicaciones, en diferentes revistas científicas, ponían en evidencia que las diferencias biológicas y el género como construcción social, influye en el proceso de las Enfermedades Cardiovasculares (ECV) y en la falta de esfuerzo terapéutico hacia

15. Pla Director de les Malalties de l'Aparell Circulatori.. Direcció General de Planificació i Avaluació 2006. Barcelona. 
las mujeres. En el decreto de creación del Plan Director y de su Consejo Asesor, tras señalar las funciones del mismo, nos dice que el desarrollo de estas funciones se hará teniendo en cuenta el género y las especificidades de salud de las mujeres para ofrecer una atención integral. En relación a la visibilidad, la base de cualquier planificación es partir del análisis de la situación para poder analizar los datos con validez, por tanto hay que tenerlos disgregados por sexo. El Plan nos presenta una descripción de la morbilidad y mortalidad disgregada por sexo pero no recoge los factores de riesgo en relación a las morbilidades señaladas disgregados por sexo y tampoco realiza un posterior análisis de estos datos buscando indicadores de género. Se limita a una descripción de datos. En un artículo de la Gaceta Sanitaria en el 2004, se publicaba:

La Cardiopatía Isquémica es un proceso multifactorial, hay evidencias de que está relacionada con determinados factores de riesgo, como la HTA, las dislipemias, la diabetes mellitus, el consumo de tabaco o el sedentarismo. Estos factores de riesgo pueden actuar de manera diferente entre mujeres y hombres, lo que señala la importancia de establecer pautas de actuación preventiva y terapéutica diferentes según el sexo y con perspectiva de género. ${ }^{16}$

En 1991 en la Revista Española de Cardiología publicaba un artículo titulado «Supervivencia en los 10 años de una cohorte de 736 pacientes con un primer infarto de miocardio: ¿Diferencias entre sexos?» En el que apuntaba diferencias que en estudios posteriores se han evidenciado, como la mayor mortalidad de las mujeres en la fase aguda asociado entre el grado de Killip y el sexo, presentando las mujeres peores puntuaciones que los hombres; así como la relación de una peor situación basal en relación a los factores de riesgo coronario, en el estudio presentado, la hipertensión arterial y la diabetes era más frecuente entre las mujeres ${ }^{17}$. En una publicación del Journal of the American Medical Association (JAMA). 1998, llegaban a la conclusión de que: «En este estudio de población, las mujeres experimentan más letalidad y gravedad en el primer infarto agudo de miocardio que los hombres, independientemente de la comorbilidad, la edad, anterior o la angina de pecho.»

«La tasa de mortalidad tras un primer infarto es mayor en las mujeres. A los 28 días después de haber sufrido el infarto más del 18\% de las mujeres

16. Rohlfs i, García M, Gavaldà L, Medrano M J, Juvinyà D, Baltasar A.»Genero y cardiopatía isquèmica».Gaceta Sanitaria, 18(2004), pp 54-64.

17. Marrugat J SAla J, Ribalta A. et al. «Supervivencia a los 10 años de una cohorte de 736 pacientes con un primer infarto de miocardio: ¿Diferencias entre sexos?». Revista española de Cardiologia. 44 (1991), pp.297-305.

Feminismo/s 18, diciembre 2011, pp. 135-154 
morían frente un $8 \%$ de los hombres y 6 meses después casi un $26 \%$ de las mujeres había muerto frente a un $12 \%$ de los hombres». ${ }^{18}$

Las mujeres tardan más en acudir al hospital, y tardan más en ser atendidas y en recibir tratamiento trombolítico. ${ }^{19}$

En relación a la presentación de la Cardiopatia isquémica (CI), en el artículo referenciado anteriormente, «Género y Cardiopatía Isquémica» nos señala:

Las manifestaciones clínicas de la CI se han estudiado más intensamente y específicamente en el hombre que en la mujer. Así, el cuadro clínico considerado como típico en el hombre (dolor precordial desencadenado por el esfuerzo, con irradiación a la mandíbula y / o brazo, acompañado de síntomas vagales, como náuseas o vómito y disnea) no tiene por qué corresponder al de la mujer a la que tiende a definirse erróneamente como atípico o anormal.

Esta característica no se refleja en el Plan Director y puede estar relacionada con la tardanza de las mujeres al pedir atención y en la falta de esfuerzo terapéutico. En el Plan Director no aparecen datos referentes a la clase social y sexo en relación a la morbilidad y mortalidad. No todos los datos están disgregados por sexo y no se realiza el posterior análisis de los datos.

En relación a la participación de los diferentes «actores» del plan (según lo llama el Plan) la composición en relación al sexo del Equipo del Plan es $42,8 \%$ mujeres frente a $57,1 \%$ hombres, en cuanto a los colaboradores / as del Departamento de Salud las mujeres representan el 42,5\% frente a 57,4\% de hombres. En el Consejo Asesor las mujeres representan el 33,3\% frente a $66,6 \%$ hombres y algunos grupos de trabajo, sobre todo los que hace referencia en la parte de las enfermedades cardiovasculares (insuficiencia cardiaca, síndrome coronario agudo, formación en el síndrome coronario, angioplastias, código infarto) suponen un $100 \%$ de hombres.

En los grupos de trabajos de las enfermedades cerebro vasculares, en el de vasculares cerebrales el $10 \%$ son mujeres mientras que en el de rehabilitación el $71,7 \%$ son mujeres.

En las entrevistas en profundidad realizadas a las personas implicadas en la elaboración del Plan, les preguntamos si los profesionales que habían participado tenían formación en género o si se había previsto la incorporación de personas con formación en género y respondieron que no.

18. Marrugat J, Sala J, Masià R, Pavesi M, Sanz G, Valle V «Mortality Differences Between Men and Women Following First Myocardial Infarction». Journal of the American Medical Association (JAMA). 280 (1998), pp.1405-1409.

19. MARRUGAT, Jaume. SALAJJoan. ABOAL Jaime. «Epidemiologia de las enferedades cardiovasculares en la mujer». Revista Española de Cardiología. 59 (2006), pp. 264-74. 
El lenguaje utiliza el masculino para referirse a los dos sexos y la imagen que utiliza en la portada es de una persona difuminada que está corriendo. En relación a la bibliografía citada en el Plan, en el apartado de propuestas, de 6 citas 1 hace referencia al sexo / género en relación a la mortalidad del IAM, en el apartado del Mapa sanitario de 13 citas ninguna hace referencia el sexo / género. En la bibliografía general referenciada en el Plan, de 15 citaciones ninguna hace referencia al sexo/género y las enfermedades del aparato circulatorio. En relación al género como determinante en las ECV, no queda reflejado en el documento. En una de las líneas de actuación, al referirse al síndrome coronario agudo se señala la importancia de la rapidez y la coordinación en la atención ya que de esto dependerá el pronóstico y la evolución de la enfermedad. Se indica que para alcanzar estos objetivos se debe poner en marcha un proyecto, teniendo en cuenta de manera específica la perspectiva de género y señala como referencia bibliográfica del estudio REGICOR. Ningún objetivo se refiere a mejorar el tiempo entre la aparición del síntoma el pedir ayuda y la respuesta del sistema sanitario. Según las personas responsables de la elaboración del Plan, no se suministró documentación para facilitar la incorporación de la perspectiva de género a las personas implicadas en la redacción del Plan, y tampoco se incorporaron personas con formación de género para su elaboración.

En la formulación de los objetivos, no se tiene en cuenta los resultados de los datos disgregados, aunque los determinantes y factores de riesgo en relación a las ECV son diferentes en los hombres y las mujeres.

No se formulan objetivos específicos en cuanto a la búsqueda de indicadores sensibles al género. No hay ninguna propuesta para disminuir los sesgos de género con el conocimiento existente en relación a las ECV. Las mujeres siguen sin considerarse enfermas cardíacas invisibilizándose el riesgo.

\section{Discusión}

Después de haber analizado los Planes Directores del Departamento de Salud de la Generalitat de Cataluña, que en los DOGC (s) correspondientes en el artículo 2.2 nos señala:

En el desarrollo de estas funciones se hará el seguimiento y la validación del uso de la variante del género, de forma que se tengan en cuenta las especificidades de salud de las mujeres con el objeto de dar respuesta y ofrecer una atención integral ante sus necesidades.

En relación a la visibilidad no todos los datos están disgregados por sexo y las que están se quedan en una mera descripción sin el análisis posterior para poder relacionar las diferencias entre sexos en posibles factores relacionados 
con el género. En relación a la participación hay un porcentaje importante de mujeres en los equipos de los Planes Directores, cabe señalar el Plan Director de Inmigración y el Plan Director Sociosanitario en que el porcentaje de mujeres alcanza un $71 \%$, en este último la codirectora es una mujer, pero el porcentaje disminuye en los grupos de trabajo y queda reflejado las especialidades más masculinizadas (anatomía patológica, las relacionadas en cardiología,..) que no cuentan con ninguna mujer y las más feminizadas que la mayoría son mujeres como pueden ser rehabilitación, mediación, acogida, salud mental, y el grupo de infantil.

El lenguaje sigue siendo androcéntrico y en ocasiones sexista. Habría que tener en cuenta estas consideraciones en los redactados de los Planes. En relación a las imágenes que muestran los Planes (portada, folletos, trípticos,..) aparecen por igual hombres y mujeres y no se aprecian indicios sexistas.

En cuanto a la transversalidad sólo se trabaja con la IDC puntualmente, en el Plan de Inmigración, en el grupo de trabajo del cáncer del cuello uterino que también está incorporada la Red de Mujeres por la Salud y en un objetivo en relación a la violencia de género del plan de Salud mental que nombra el ICD. En relación al género como determinante no utilizan bibliografía en relación al género y el Plan Director determinado, sólo hay una referencia al Plan de las enfermedades cardiovasculares.

Los objetivos de los Planes son muy genéricos y no reflejan las diferencias descritas en el análisis de la situación.

Las personas que han intervenido en la redacción del Plan no han recibido formación en género ni se les ha proporcionado bibliografía en relación al género y el plan determinado.

Aceptamos nuestra hipótesis de partida que la voluntad política de integrar la perspectiva de género en el Sistema Sanitario Catalán, expresada en el artículo 2.2 de todos los Planes Directores, no queda reflejada en la redacción de los mismos y se traduce parcialmente en el diseño de los mismos.

\section{Referencias bibliográficas}

Agencia de investigación de la Sociedad Española de Cardiologia. Enfermedad cardiovascular en la mujer, estudio de la situación en España. Informe realizado para el Observatorio de Salud de la Mujer (OSM). Ministerio de Sanidad y Consumo. Madrid. 2008.

Amo Alonso, Mercedes. Guía para facilitar la incorporación de la perspectiva de génroen los Planes Integrals de salud. Sevilla.Consejeria de Salud. 2008.

AstelarRA, Judith, Veinte años de politicas de igualdad.Madrid.Ediciones Cátedra. 2005. 
Garcia Calvente $\mathrm{M}^{\mathrm{a}}$ del Mar, Jimenez Rodrigo Ma Luisa, Martinez Morante Emilia. Informe de revisión de guias sobre la incorporación de la perspectiva de género a las plíticas de investigación en salud. Observatorio de Salud de la Mujer (OSM). Dirección general de la agencia de Calidad del Sistema nacional de Salud. Secretaria General de Sanidad. Ministerio de Sanidad y Consumo. Escuela Andaluza de Salud Pública. 2005.

GuZMAN,Virginia.La institucionalidad de género en el estado: nuevas perspectivas de análisis.Chile.CEPAL,Série Mujer y Desarrollo,núm 3, 2009.

Generalitat de Catalunya, Pla de Salut de Catalunya a l'horitzó 2010. Departament de Salut. Barcelona: 2008.http://www.gencat.cat/salut/depsalut/html/ca/ plasalut/index.html, última consulta 1-3-2011.

Generalitat de Catalunya. Pla de polítiques de dones del govern de la Generalitat de Catalunya 2008-2011 Institut Català de les dones (ICD). Barcelona 2008.

Generalitat de Catalunya. Pla Director d'Immigració en l'Àmbit de la Salut. Direcció General de Planificació i Avaluació Barcelona. 2006.http://www.gencat. cat/salut/depsalut/html/ca/dir2154/index.html. Consultado 25-9-2010.

Generalitat de Catalunya. Pla Director Socioanitario. Direcció General de Planificació i Avaluació.Barcelona 2006.http://www.gencat.cat/salut/depsalut/html/ ca/dir2154/index.html. Consultado 25-9-2010.

Generalitat de Catalunya. Pla Director d'Oncologia. Direcció General de Planificació i Avaluació Barcelona 2006. http://www.gencat.cat/salut/depsalut/html/ ca/dir2154/index.html. Consultado 25-9-2010.

Generalitat de Catalunya. Pla Director de les Malalties de l'Aparell Circulatori.. Direcció General de Planificació i Avaluació.Barcelona 2006.http://www.gencat.cat/salut/depsalut/html/ca/dir2154/index.html. Consultado 25-9-2010.

MARRUGAT J SALA J, Ribalta A. et al. «Supervivencia a los 10 años de una cohorte de 736 pacientes con un primer infarto de miocardio: ¿Diferencias entre sexos?». Revista española de Cardiologia. 44 (1991), pp. 297-305.

Marrugat J, Sala J, Masià R, Pavesi M, SAnz G, VAlle V «Mortality Differences Between Men and Women Following First Myocardial Infarction». Journal of the American Medical Association (JAMA).280 (1998), pp. 1405-1409.

MARRUGAT, Jaume. SALA, Joan. ABOAL Jaime. «Epidemiologia de las enferedades cardiovasculares en la mujer». Revista Española de Cardiología. 59 (2006), pp. 264-74.

LEY 3/2007, de 22 de marzo, para la igualdad efectiva de mujeres y hombres. (BOE n ${ }^{\circ} 71$ de 23-3-2007)

ORGANIZACIÓN MUNDIAL DE LA SALUD (OMS). Integración de la perspectiva de género en la labor de la OMS. Ginebra 2002. http://whqlibdoc.who.int/ hq/2002/a78343_spa.pdf 
Peiró R, Ramon N, Álvarez-Dardet C, Colomer C, Moya C, Borell C, i altres. «Sensibilidad de género en la formulación de planes de salud en España: lo que pudo ser y no fue. Gaceta Sanitaria. 2004; 18(2).

ROHLfs I, Garcia M, GaVAldÁ L, Medrano MJ, Juvinyà D, BALtasar A.»Genero i cardiopatía isquèmica».Gaceta Sanitaria, 18(2004), pp 54-64.

Ruiz Cantero Ma Teresa, Papí Galvez Natalia.Guía de estadísticas de salud con enfoque de género. Análisis de internet y recomendaciones. Universidad de Alicante. 2007.

Ruiz-Czntero Maria Teresa, CARrasco Portiño Mercedes, ArTAzCoz Lucía. «Logros y retos de género de la Encuesta Nacional de Salud de 2006: análisis de los cuestionarios de adultos y hogar». Gaceta Sanitària, 25(1), pp6-12. 2011.

SIPI, Remei, ARAYA, Mariel. Les dones migrades.Generalitat de Catalunya.Institut Català de les Dones. Barcelona. Quaderns de l'Institut 5. (2007).

VElASCO ARIAS Sara. Recomendaciones para la práctica del enfoque de género en programas de salud. Observatorio de Salud de la Mujer (OSM). Dirección general de la agencia de Calidad del Sistema nacional de Salud. Secretaria General de Sanidad. Ministerio de Sanidad y Consumo Madrid 2008.

VELASCO ARIAS Sara. Informe sobre netodologia y determiantes psicosociales de género en enfermedadfes cardiovasculares de la Comunidad Valenciana Dirección General de salud pública Conselleria de Sanitat. Generalitat Valenciana, 2010. 